\title{
Ser dos en el aula: las parejas pedagógicas como estrategia de co-enseñanza inclusiva en una experiencia de formación inicial del profesorado de secundaria
}

\author{
Manuel Cotrina García, Mayka García García, Ester Caparrós Martín \\ Departamento de Didáctica. Universidad de Cádiz
}

\section{RESUMEN}

Este trabajo aborda la "pareja pedagógica" como estrategia didáctica innovadora de corte inclusivo, que caracteriza una modalidad de enseñanza colaborativa (co-enseñanza). Esta estrategia requiere la implicación, intencional y voluntaria de dos profesores en todos los aspectos vinculados a la docencia: planificación, evaluación y, singularmente, durante el desarrollo de las sesiones de clases. Inicialmente se revisa la literatura sobre el tema de estudio, de cara a documentar su potencial en la mejora del proceso didáctico, e ilustrar sus implicaciones para el desarrollo profesional docente; asimismo, se centra el eje de análisis en la educación inclusiva. En un segundo momento, se presentan los resultados de un proyecto de innovación docente cuyo eje central es la puesta en acción de esta estrategia por un grupo de docentes de la Universidad de Cádiz, en diversas titulaciones vinculadas a la formación inicial del profesorado. En concreto, se presenta la visión particular del alumnado de la asignatura "Procesos y Contextos Educativos", del Máster en Formación del Profesorado de Secundaria. Es a través de la voz del alumnado que el modelo de co-enseñanza, llevado a cabo en esta experiencia con las "parejas pedagógicas", adquiere valor pedagógico y se sitúa como motor para la proyección hacia su práctica futura.

Palabras Clave: co-enseñanza, pareja pedagógica, educación inclusiva, formación inicial del profesorado.

\section{Two in the classroom: pedagogical partners as an inclusive co-teaching strategy in preservice secondary teachers}

ABSTRACT

This work approaches the "pedagogical partnerns" as an innovative didactic strategy of inclusive orientation, which characterizes a modality of collaborative teaching (co-teaching). This strategy requires the intentional and voluntary involvement of two teachers in all aspects related to teaching: planning, assessment and, in particular, during the development of class sessions. Initially the literature on the subject of study is reviewed, in order to documents its potential in improving the didactic process, and to illustrate its implications for teacher professional development; likewise, the analysis focuses on inclusive education. Secondly, we presents the results of a teaching innovation project whose central axis is the implementation of this strategy by a group of teachers of the University of Cadiz, in various degrees related to the preservice teachers. Specifically, we present the students' particular view on the subject "Processes and Educational Contexts" of the Master in Secondary Teacher Training. It is through the voice of the students that the co-teaching model, carried out in this experience with the "pedagogical partners", get its pedagogical value and is placed as a motor so that the students can project it towards its future practice.

Keywords: co-teaching, pedagogical pair, inclusive education, initial teacher training.

\section{A modo de introducción}

Una de las cuestiones más importantes que se abordan dentro del ámbito de la formación de los profesionales de la educación, tienen que ver con las reflexiones y los análisis que ponen en cuestión la calidad de los procesos de enseñanza y aprendizaje; así como su repercusión en la mayor o menor calidad de los resultados que se obtienen. Lo que a continuación presentamos guarda relación precisamente con esto. Se trata de una indagación en relación con una innovación en la formación inicial del profesorado a partir del desarrollo de parejas pedagógicas, una estrategia didáctica que se adscribe a la co-enseñanza.

La presentación de la co-enseñanza como estrategia pedagógica con alto valor es algo que está presente en la mayoría de la literatura en relación con la enseñanza colaborativa. Ya desde los años 90, es posible identificar distintos trabajos que presentan 
y/o analizan experiencias desde dos focos temáticos fundamentales. De un lado, desde su potencial como estrategia de apoyo en el aula (Bauwens, Hourcade \& Friend, 1998; Friend, Cook, Hurley-Chamberlain \& Shamberger, 2010) orientada a ofrecer una respuesta educativa más ajustada al alumnado que precisa una atención particular, ya sea puntual o sostenida en el tiempo. De otro, como estrategia de andamiaje en el desarrollo profesional del profesorado, partiendo de la reflexión compartida sobre la propia práctica que ayuda a transformar el currículum (Duran \& Miguel, 2003; Kroegera, Embury, Cooper, Brydon-Miller, Laine \& Johnson, 2012).

Teniendo en cuenta que estos son los enfoques predominantes en la co-enseñanza, este trabajo resulta novedoso, en el sentido de que procura, desde el inicio, la fusión armónica de ambas líneas, bajo la mirada de lo que consideramos ofrece una educación inclusiva como una forma de ser, estar y vivir en educación (García \& Cotrina, 2015). Esto conlleva re-imaginar los procesos y los contextos educativos enriqueciéndolos de experiencias llenas de significatividad para quienes participan en ellas. Asumimos, pues, la co-enseñanza como un proceso de apoyo colaborativo al aula (Parrilla, 2003), donde los docentes llevan a cabo, como parejas pedagógicas, un proceso formativo inclusivo (Moriña \& Parilla, 2006) orientado a la optimización de la enseñanza y del aprendizaje.

Así pues, centramos la puesta en acción de las parejas pedagógicas dentro de un contexto particular de formación inicial del profesorado, el Máster de Profesorado en Enseñanza Secundaria Obligatoria y Bachillerato, Formación Profesional y Enseñanza de Idiomas; donde consideramos fundamental apostar por una formación profesionalizadora, que posibilite el desarrollo de una identidad profesional (Bolívar, 2007). Como apunta Marínez Bonafé (2010), donde el aprendizaje del oficio docente ayude a sistematizar la práctica. De manera que es este el propósito que justifica este trabajo.

\section{Marco conceptual}

Para situar el discurso, sostenemos que la co-enseñanza puede identificarse bajo el paragüas de otras denominaciones como co-docencia (co-teaching), enseñanza colaborativa, enseñanza en equipo (Chanmugam, \& Gerlach, 2013), cátedra compartida o co-enseñanza (Suárez-Díaz, 2016), pareja pedagógica (Bekerman, \& Dankner, 2010; Rodríguez \& Grilli, 2013) o pareja educativa (Hoyuelos, 1994; Salamanca, 2015). En este caso apostamos por nombrar la estrategia didáctica que presentamos desde la definición de pareja pedagógica; ya que consideramos que el el concepto de "pareja" guarda un componente simbólico que se sostiene en una manera determinada de establecer la relación entre los docentes que la conforman, esta es, el respeto como reconocimiento mutuo (Bekerman, \& Dankner, 2010). La pareja pedagógica supone elegir-se, a veces sin conocer-se, para descubrir-se; hay en ello una intencionalidad que compromete a los docentes a aceptar que ha de existir una relación de confianza y de reconocimiento de autoridad a la hora de construir conocimiento y negociar las relaciones de poder como características propias de la co-enseñanza (Bruffee, 1999).

Por otro lado, la literatura en torno a ésta nos remite al ámbito de la atención a la diversidad, donde se ha desarrollado como una estrategia de gran interés que re-posiciona en el aula al profesorado de educación especial en el contexto de una educación inclusiva (Bauwens, Hourcade \& Friend, 1998). Y es que una buena parte del desarrollo de la co-enseñanza ha venido de la mano de mejoras a la atención educativa al alumnado que presenta necesidades educativas especiales. Sin embargo, Die- ker \& Murawski (2003) nos advierten de cómo la práctica de la co-ensenañza surge, en primera instancia, como una estrategia docente en la enseñanza ordinaria, desde el mismo sentido optimizador por el que este trabajo apuesta. De hecho, autoridades en la materia, se esfuerzan por enfatizar las características inclusivas de esta práctica (Stainback \& Stainback, 1999), apostando por de-construir una visión que se ha ido consolidando en los últimos 15 años en relación a la co-enseñanza, que se centra en orientar exclusivamente dicha estrategia para el apoyo del alumnado que presenta una discapacidad; lo que la limita a ser una herramienta al servicio de la integración educativa. En esta dirección apunta también Dieker (2013), quien afirma que "no importa el tipo de co-enseñanza que uses, en las clases inclusivas, el modelo que se utilice está dirigido a atender las necesidades de todos los estudiantes y no solamente a las de aquellos que presentan discapacidad" (p.66).

Desde una orientación inclusiva, la co-enseñanza emerge como una actividad compleja desde la que se repiensan las prácticas educativas. Pugach y Johnson (2002) amplían e identifican, al menos, cuatro funciones de la pareja pedagógica en el aula: facilitadora, de apoyo, informativa y prescriptiva. En esta línea, Dieker \& Murawski (2003) y Chanmugam \& Gerlach (2013) aluden a que la co-enseñanza se define por la articulación de la relación pedagógica intencional entre dos profesores del mismo status, distinguiendo esta práctica de otras en la que están presentes personas voluntarias o asistentes y superando la visión clásica de profesor generalista y profesor de educación especial (o especialista de la orientación); así como hacen notar que ese trabajo conjunto no se puede limitar a acciones esporádicas, sino que ha de articularse en el diseño, el desarrollo y la evaluación del currículum. Esta concepción compleja (y cambiante) se ha puesto de manifiesto en la definición progresiva de distintos tipos de co-teaching, como puede apreciarse en la tabla posterior, donde las mismas autoras reflejan nuevas posibilidades de acción didáctica.

Todos estos tipos de co-teaching tienen en común la esencia inclusiva de esta enseñanza (Friend, \& Cook, 2007) que implica, necesariamente, la presencia de dos personas que ejercen papeles definidos desde un liderazgo compartido, redefinen su posición de autoridad y sus roles. Así, reconociéndose en un mismo status, participan del diseño, desarrollo y evaluación de la propuesta didáctica; contribuyen colaborativamente al desarrollo de una comunidad más acogedora, donde el etiquetaje se diluye (Durán \& Miguel, 2003); en una clase heterogénea donde se valoran las diferencias de estudiantes y de profesores (Keefe \& Moore, 2004) porque existe una relación de escucha mutua.

Dentro del contexto de la Educación Secundaria, la co-enseñanza fue vista como una posibilidad para reformar lo que pasaba en las aulas norteamericanas de los 90 (Cook \& Friend, 1995), como ya había ocurrido años atrás en la experiencia inglesa. En este mismo contexto, Dieker \& Murawski (2003) la presentan como una acción docente que facilita: (i) el abordaje de los contenidos a través del aprendizaje cooperativo, así como la articulación de procesos didácticos de corte socio-constructivista; (ii) que el profesorado considere estrategias alternativas para evaluar; y (iii) la atención a las necesidades individuales de los estudiantes.

Para finalizar este recorrido del estado de la cuestión en relación con la co-enseñanza, podemos señalar que existen trabajos que revisan las principales aportaciones de la estrategia, entre los que cabe destacar los de Murawski \& Lee (2001) y Scruggs, Mastropieri \& McDuffiel (2007). Las primeras realizan un meta-análisis de investigaciones de orientación cualitativa, mientras que los segundos lo hacen de aquellas de orientación cuantita- 
Tabla 1. Evolución de tipos de co-enseñanza

\begin{tabular}{|c|c|c|c|}
\hline Tipos de co-enseñanza & $\begin{array}{l}\text { Cook \& } \\
\text { Friend, } \\
\text { (1995) }\end{array}$ & $\begin{array}{l}\text { Cook } \\
(2004)\end{array}$ & $\begin{array}{l}\text { Friend et } \\
\text { al (2010) }\end{array}$ \\
\hline $\begin{array}{l}\text { One Teach, One Observe } \\
\text { Co-enseñanza de observación } \\
\text { [uno enseña, otro registra lo que ocurre] }\end{array}$ & & & $\bullet$ \\
\hline $\begin{array}{l}\text { One Teach, One Assist } \\
\text { Co-enseñanza de apoyo } \\
\text { [uno enseña, otro apoya a estudiantes que lo requiere] }\end{array}$ & $\bullet$ & $\bullet$ & $\bullet$ \\
\hline $\begin{array}{l}\text { Parallel Teaching } \\
\text { Enseñanza en grupos simultáneos } \\
\text { [clase divida en dos y cada profesor se dedica a un grupo] }\end{array}$ & $\bullet$ & $\bullet$ & $\bullet$ \\
\hline $\begin{array}{l}\text { Rotation Teaching } \\
\text { Co-enseñanza de rotación entre grupo } \\
\text { [existen distintos equipos de trabajo y el profesorado va acudiendo a los } \\
\text { distintos equipos] }\end{array}$ & & & $\bullet$ \\
\hline $\begin{array}{l}\text { Complementary Teaching } \\
\text { Co-enseñanza Complementaria } \\
\text { [profesor-a desarrolla la sesión y el-la otro-a apoya con comentarios, } \\
\text { ejemplos, etc.] }\end{array}$ & & $\bullet$ & $\bullet$ \\
\hline $\begin{array}{l}\text { Station Teaching } \\
\text { Co-enseñanza en estaciones } \\
\text { [división de la clase en } 2 \text { grupos, cada profesor muestra el mismo tema a } \\
\text { un grupo y luego al otro] }\end{array}$ & $\bullet$ & $\bullet$ & $\bullet$ \\
\hline $\begin{array}{l}\text { Alternative Teaching } \\
\text { Co-enseñanza Alternativa } \\
\text { [un profesor es responsable de un grupo más amplio y el otro de otro } \\
\text { grupo más pequeño] }\end{array}$ & $\bullet$ & $\bullet$ & $\bullet$ \\
\hline $\begin{array}{l}\text { Team Teaching } \\
\text { Co-enseñanza en equipo } \\
\text { [la sesión supone un diálogo a dos voces]. }\end{array}$ & $\bullet$ & $\bullet$ & $\bullet$ \\
\hline
\end{tabular}

Elaboración propia a partir de los trabajos de A Cook, L. \& Friend, M. (1995), Cook (2004) y Friend, M., Cook, L., Hurley-Chamberlain, D. y Shamberger (2010)

tiva. Por su parte, los trabajos de Friend (2008) y Mastropieri et al. (2005) permiten ilustrar los principales focos que centran la atención sobre el tema:

- El impacto y la eficacia de la co-enseñanza sobre el aprendizaje de los estudiantes.

- Las percepciones de estudiantes y profesorado sobre las prácticas de co-enseñanza.

- Las implicaciones de la distribución de roles y las relaciones entre docentes.

- La planificación y programación, organización didáctica de espacios, tiempos y agrupamientos del alumnado y la evaluación.

En el trabajo que se presenta a continuación confluyen algunos de los focos de indagación antes mencionados.

\section{Aspectos Metodológicos}

El objetivo de este estudio ha sido conocer la percepción e interpretación que los estudiantes, de dos grupos-clase de una misma materia, tienen sobre el diseño y la puesta en práctica de la actividad docente en dicha asignatura, a través de la propuesta didáctica que hemos denominado la pareja pedagógica. El trabajo que se presenta se adscribe ${ }^{1}$ a un proyecto de innovación educativa articulado en distintas titulaciones de Educación de la Universidad de Cádiz, orientado a: 1) Mejorar la atención a la diversidad del alumnado en aulas universitarias diversificadas,
2) El desarrollo de equipos docentes en los títulos y con ello, la mejora de los procesos de enseñanza-aprendizaje en los mismos, y 3) La institucionalización de la innovación en confluencia con otras innovaciones previas.

\section{Proceso de recogida de información}

La población invitada como informante en el estudio fue todo el alumnado de dos grupos que durante el curso 16-17, participó en la asignatura Procesos y Contextos, materia obligatoria perteneciente al módulo común del Máster en Profesorado de Enseñanza Secundaria Obligatoria y Bachillerato, Formación Profesional y Enseñanza de Idiomas (MAES). A la invitación respondieron un total de 18 estudiantes, 8 del grupo " $\mathrm{A}$ " y 10 del grupo " $\mathrm{D}$ ". Para recabar la información y conocer la opinión de los estudiantes, se solicitó la realización de un relato narrativo sobre sus puntos de vista y la experiencia que desarrollaron los docentes implicados como "pareja pedagógica" durante la docencia de la asignatura. El proceso de recogida de información se llevó a cabo una vez que la asignatura había finalizado. Cabe resaltar que desde el inicio de la misma se había presentado al alumnado dicha estrategia didáctica como parte del proceso de enseñanza y aprendizaje diseñado y desarrollado por el profesorado de ambos grupos y se explicó su sentido en su propio proceso formativo. Además, para poder recabar datos por distintas vías (p.e. fotografía) y para hacer un uso científico de esta información, se solicitó el permiso previo de los estudiantes. 
Análisis de datos

Dado que los docentes conformaban dos parejas pedagógicas distintas, el análisis de la información se realizó, en primer lugar, de manera independiente para cada grupo, lo que permitiría la emergencia de categorías de análisis; posteriormente, se analizaron conjuntamente, triangulación de fuentes.
El análisis de la información se inició atendiendo a tres categorías teóricas: Valoración general, Aspectos positivos/fortalezas y Aspectos conflictivos/debilidades. A partir la primera reducción de información emergieron tres dimensiones conceptuales de análisis que pueden apreciarse en la siguiente figura.

Para mantener el anonimato de las fuentes, se codificaron los informantes de la siguiente manera: cada "estudiante" mediante

\begin{tabular}{|l|}
\hline $\begin{array}{l}\text { Valoración general de } \\
\text { la experiencia }\end{array}$ \\
\hline - Miedos \\
- Grado de satisfacción \\
- Impresiones \\
generales \\
-Innovación
\end{tabular}

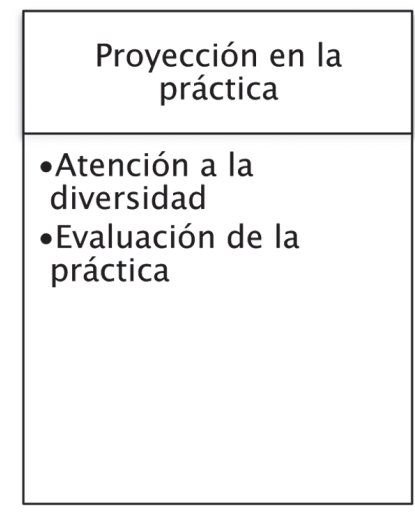

Figura 1. Dimensiones y categorías emergentes. la letra "E" seguido del número que le ha sido asignado dentro de cada grupo, y "GA" y "GD" que permite distinguir la adscripción de los estudiantes al grupo " $\mathrm{A}$ " $\mathrm{o}$ " $\mathrm{D}$ " respectivamente. Por otro lado, para garantizar el anonimato del profesorado que fue nombrado expresamente en la información aportada por el alumnado se utilizó la inicial del nombre y adscripción al grupo.

\section{Resultados}

¿Cómo valora el alumnado la experiencia?

El sentido que el alumnado otorga a la experiencia es una de las cuestiones que consideramos importantes a la hora de valorar esta propuesta de parejas pedagógicas. Significa que tenía que posicionarse críticamente, emitiendo un juicio reflexivo, sobre aquello que considera importante y valioso para su proceso de aprendizaje como docente. Al evaluar la experiencia, los estudiantes muestran su capacidad para pensar desde dos planos que, aunque son dispares, se complementan. El primero, el de la propia experiencia vivida como estudiantes que experimentan la apuesta por la pareja pedagógica en su aula. El segundo, las posibilidades formativas que ofrece dicho escenario para construir un tipo de pensamiento pedagógico docente más ligado a procesos innovadores.

Es por ello que, al comienzo de la experiencia, explicitamos a nuestro alumnado la propuesta didáctica que estábamos llevando a cabo y pudimos observar como algunos mostraban juicios previos que se sostenían en un inicial miedo a lo desconocido. En este sentido, los estudiantes reconocen en sus relatos que les resultaba una propuesta un tanto extraña (en cuanto que poco común), y mostraban ciertas reticencias al planteamiento. Sobre todo, desconfianza ante aquello que "lo nuevo" les ofrecía, sentimiento que guardaba relación con una primera sensación de que la gestión de aula se podría ver afectada por dinámicas más desordenadas.

Al comienzo de las mismas creí que la idea de llevar a cabo las clases mediante "parejas pedagógicas" podría resultar un poco caótica o desordenada (E1GA).
Sin embargo, parece que, a tenor de lo expresado en los relatos, los miedos se fueron disipando a lo largo de la nueva acción docente. De hecho, prácticamente la totalidad de los estudiantes de los que se ha recabado información consideran y valoran como muy positiva la experiencia en términos de satisfacción y utilidad formativa, como puede ilustrarse a través de muchas de las citas que hemos obtenido y en las que se repite esta idea satisfacción positiva constantemente.

Mi valoración tras finalizar las clases de PyC resulta ser bastante satisfactoria. (E1GD)

Las parejas pedagógicas resultan una herramienta muy útil para poder trabajar de una forma más intensa y rica en cuanto a formación se refiere. (E3GA)

Si nos detenemos en este alto grado de satisfacción manifestado podemos observar como tiene que ver, según la opinión del siguiente alumno, con el hecho de que les resulte una experiencia de aprendizaje de la que tienen la oportunidad de sacar más partido; en el sentido de que les sirve de espejo sobre el que pensarse como fututos docentes.

Desde mi punto de vista, creo que el hecho de que la asignatura haya sido impartida por dos docentes es más enriquecedor para nosotros como futuros docentes. (E5GA)

Y es que hay que tener en cuenta que, en el contexto donde se desarrolla la experiencia, un máster profesionalizador que habilita para la función docente, el alumnado descubre otras formas de ser y estar en la docencia, distintas a las que ha vivido como estudiante. Y eso además les capacita para poner en cuestión muchas de las creencias que traen a clase al principio y reformularlas viviendo en primera persona nuevas maneras de enseñar y aprender. De hecho, hay alumnos que reconocen lo novedosa que le resulta esta experiencia vivida, ya que carecen de referentes y experiencias previas; en este caso, lo entienden como un elemento motivador que les permite abrir sus posibilidades formativas y les lleva a reflexionar sobre otras vividas. 
El contar con dos docentes en el aula, impartiendo a la misma vez la clase, ha sido muy novedoso para mí, puesto que como estudiante universitaria nunca lo había experimentado, ni me lo había planteado. (E10GD)

Otro elemento que cabe destacar en esta primera valoración general es cómo el propio alumnado reconoce, en el ejercicio de esta práctica docente, un componente de innovación educativa.

Mi valoración acerca de las parejas pedagógicas es muy positiva y creo que nos ha abierto una puerta más a metodologías innovadoras. (E7GA)

Este comentario de la alumna muestra cómo el hecho de poder pensar la mejora la práctica educativa desde la propia experiencia les lleva a valorar la propuesta de las parejas pedagógicas como una cuestión que vas más allá de una novedad (Carbone11, 2001). Esto tiene que ver con cómo el contexto formativo en el que se encuentran les ayuda a darse cuenta que la innovación educativa no es solo un cambio per se, sino que es un proceso de enriquecimiento para todos los implicados, el alumnado y el propio profesorado, descubriendo una perspectiva docente reflexiva y, a veces, incluso crítica.

Desde mi punto de vista el trabajo por parejas pedagógicas realizado en la asignatura de Procesos y Contextos ha sido un elemento innovador y enriquecedor, tanto para los alumnos como para los profesores. (E8GA)

Tras este primer acercamiento a las opiniones emitidas por los estudiantes en torno a la valoración global de la experiencia, pasaremos a continuación, a profundizar en otra dimensión que se encuentra estrechamente ligada a la satisfacción general y al sentimiento emitido por el alumnado, y que tiene que ver con el valor pedagógico que le otorgan a la propuesta didáctica.

\section{El valor pedagógico de la experiencia}

Más allá de una valoración positiva o satisfactoria de la experiencia, los relatos de los estudiantes nos han permitido interpretar en profundidad el valor pedagógico que en sí misma tiene la experiencia. Con valor pedagógico nos referimos a la capacidad formativa que tiene el hecho de vivir la docencia de otra forma distinta a la que los estudiantes vienen experimentando en su vida académica dentro de la Universidad. En este sentido, cabe mencionar como el alumnado destaca las posibilidades que ofrece el que hecho de que haya dos docentes a la vez en el aula interaccionando de manera intencional. Destacan como eso permite la incorporación de más de un punto de vista sobre los contenidos o aspectos que en cada momento se estén tratando en la asignatura.

Por otro lado, la pareja pedagógica hace posible que haya distintos puntos de vista por parte del profesorado. (E5GD)

Y ello es un elemento de valor en especial por tener la oportunidad de aproximarse al conocimiento desde perspectivas distintas y complementarias. De hecho, señalan que la experiencia resulta enriquecida cuando existen planteamientos conceptuales y metodológicos distintos entre quienes conforman la pareja pedagógica.

Ahora bien, considero que esta metodología es interesante y enriquecedora para el alumno si estos dos profesores no necesariamente aportan la misma visión de los temas, es decir, si cada uno de ellos aporta diferentes perspectivas del mismo tema, enriqueciendo así la experiencia. (E10GD).

Desde nuestro planteamiento, consideramos que esta forma de aproximarse al conocimiento ofrece un andamiaje que posibilita el desarrollo del pensamiento crítico, es decir, que cada sujeto pueda ir construyendo un pensamiento propio a la luz de las experiencias vividas; siendo esto un requisito fundamental para el ejercicio de la docencia.

Por otro lado, el alumnado señala que la pareja pedagógica es también una oportunidad para el desarrollo profesional docente. Estos ven cómo la estrategia de compartir el aula permite que ambos docentes se enriquezcan gracias a que existe un aprendizaje y un acompañamiento mutuo. Señalan como en dicha experiencia los docentes que trabajan juntos se observan, se formulan críticas, reflexionan en común y con ello avanzan profesional y personalmente y lo valoran como un ejercicio de mutuos aprendizajes.

Entre vosotros podéis aportaros críticas constructivas, consejos, etc., y ver la forma de dar clase que tiene otro profesor y otras formas de hacer las cosas. En definitiva, aprender el uno del otro. (E2GA)

Además, una pareja pedagógica de profesores complementarios, que trabajan de manera armónica, supone una oportunidad para el alumnado de vivir en una única experiencia didáctica, distintas formas de hacer (propuestas metodológicas) de las que nutrir su bagaje experiencial y sobre las que reflexionar.

Por parte del profesor he sentido un tipo de clases como más reflexivas y de argumentación, para sacar nuestra forma de ver las cosas y que nos cuestionáramos todo (...) mientras que por parte de la profesora he visto unas clases más dinámicas, con supuestos prácticos en los que teníamos que meternos en el papel para entender que es lo que estábamos viendo y mucho trabajo en equipo. Me parece que la fusión de ambos tipos de actividades son las que han hecho de estas clases algo diferente y especial.. (E5GA)

Y es que la práctica de la pareja pedagógica no significa anular estilos docentes, sino aportar la esencia de lo que cada uno es a un acto de enseñanza aprendizaje en común, para transformarlo en algo distinto y mejor que lo que cada uno es por separado. En este sentido, el alumnado percibe, por ejemplo, que las parejas pedagógicas permiten mejorar la gestión de aula, porque permite interpretar lo que acontece en los procesos y contextos educativos desde opciones diversas.

Cuatro ojos ven más que dos ojos, y son dos puntos de vista. Lo que a un profesor le puede pasar desapercibido en el aula, puede que a otro no; o bien, que la misma situación sea interpretada desde dos puntos de vista distintos. (E2GA)

La pareja pedagógica establece un tipo de relaciones cercanas con el alumnado, al que percibe como más accesible. Ello es relevante el contexto de una asignatura de corta duración, conde las relaciones se construyen solo en 3 meses, a través de 12 sesiones de clase.

Desde el punto de vista como alumno, considero que esto ha favorecido la relación con los docentes, pues quizás no solo tienes como referencia a una sola persona y no solo se 
somete la realización de los trabajos y desarrollo de la clase a un solo juicio y valoración. (E6GA)

Otro aspecto que destacan es que permite incorporar una segunda voz a las explicaciones o aclaraciones sobre la materia, lo que mejora la comprensión del objeto de estudio.

El hecho de tener dos profesores a la vez en el aula da mucho juego puesto que presenta dos puntos de vista distintos, dos formas de expresión que permite aprender doblemente. (E2GD)

Y ello acontece en dos sentidos, dos oportunidades de comprensión para el alumno, pero también dos oportunidades de ser comprendido.

Además, en las intervenciones de los alumnos en clase, ya sea para dar la opinión sobre algo o plantear alguna duda, puede ayudar también, ya que a veces el profesor puede que no termine de entender lo que está planteando el alumno, y no conteste de manera adecuada. Sin embargo, al disponer de dos profesores, la probabilidad de que uno de los dos sepa solucionar correctamente lo que el alumno plantea es mayor. (E5GD)

Los estudiantes valoran que esta estrategia supone una situación didáctica más rica y estimulante que la que se genera con la presencia de un solo profesor en el aula, así como que favorece la implementación de propuestas más dinámicas, existiendo un intercambio de roles continuo en el aula que propicia este tipo de propuestas.

Desde mi punto de vista como estudiante creo que la presencia de dos profesores hace la clase mucho más dinámica, activa, amena e interesante ya que hay, en algunos casos, opiniones y aportaciones diferentes desde la perspectiva del profesor. Es, en mi opinión, una situación enriquecedora para el alumno. (E6GD)

Otro de los aspectos positivos que los estudiantes encuentran tiene que ver con la disminución de la ratio profesor-alumno del grupo clase y su incidencia en la mejora de la atención que se presta a los propios estudiantes.

El primero y más obvio es el de reducir la ratio profesor-alumno. En clase, cuando hemos trabajado en grupo, disponer de dos profesores ha facilitado y agilizado nuestra tarea como alumnos, pues las dudas se resolvían con mayor rapidez. (E5GD)

En este sentido, cabe destacar que el alumnado siente que se satisface su necesidad y/o demanda de atención educativa. Además, siendo consciente de las implicaciones que para los propios docentes tiene su desarrollo, en términos de esfuerzo y compromiso, valora el ejercicio de la pareja pedagógica desde una óptica de la pedagogía del cuidado (Noddings, 2002; Sierra, Caparrós y Martín, 2016).

También nos sentimos importantes, porque realmente es más trabajo para vosotros y sin embargo os tomáis la "molestia" de estar ahí de dos en dos. Yo siento que hay más personas atentas a mí, o que si uno no ve algo, el otro puede que sí lo vea. (E2GA)

Los alumnos se sienten apoyados y depositan esa confianza en ambos profesores. (E6GA)
Otro aspecto revelador que emerge del análisis de las respuestas son sus posibilidades en relación con la atención a la diversidad (Walther-Tomas, Bryant y Land, 1996). Este potencial ha sido reconocido en la práctica por los estudiantes afirmando cómo ser dos docentes es un plus que permite cuidar las distintas necesidades del alumnado; docentes que a la vez que revelan estar preparados para enseñar juntos, son y se muestran diferentes.

Aportando mi valoración personal a cerca de las parejas pedagógicas en el aula considero que es una medida apropiada que pretende atender a la diversidad y de la que, además, los alumnos se benefician al recibir dos inputs coordinados, pero distintos. (E7GD)

Proyección en su propia práctica: las parejas pedagógicas en Educación Secundaria.

Es un rasgo característico de esta asignatura y del diseño didáctico del MAES, el hacer del propio proceso didáctico un ejercicio de aprendizaje en relación la práctica profesional, dicho de otra manera, llevar la teoría didáctica a la práctica. En la vivencia de esa práctica descubrimos también las posibilidades de acción. El abordaje de parejas pedagógicas en el contexto del MAES no es anecdótica, responde a una intencionalidad formativa en la propia estrategia a través de la acción.

Este proceso de recogida de información del alumnado, como acto reflexivo, se ha configurado como un proceso intencionado en torno al modus docendi de nuestro alumnado, o lo que es lo mismo, una oportunidad para pensar en la proyección de esta estrategia en la docencia en el contexto de la Educación Secundaria, proceso para el que se están formando. Han destacado el valor de la pareja pedagógica desde tres orientaciones: como estrategia para atender a la diversidad, para desarrollar el currículum y desde la idea de apoyo educativo. Todo ello, rescatando la idea de que ser pareja pedagógica va más allá de una anécdota didáctica puntual.

Han apuntado, por ejemplo, como las parejas pedagógicas se erigen como una estrategia que asumirían como propia de cara a responder a la diversidad en sus aulas, porque les permitiría atender mejor al alumnado, duplicando efectivos.

En mi opinión en una clase tan grande como ha sido la nuestra ha sido útil contar con dos docentes en el aula. De cara a nuestra vida profesional lo veo aún más interesante porque no sólo contamos con clases demasiado numerosas, además con una diversidad de alumnos que es inviable que un solo profesor pueda atender a todos. (E9GD)

En otros casos, han sido capaces de ver proyectada la estrategia en el desarrollo de sus prácticas curriculares y han comprendido, la profundidad de la misma y la complejidad de diseño que hay detrás, más allá de que se convierta en la presencia anecdótica de dos docentes en un mismo espacio, especialmente como una pedagogía inclusiva.

En mi caso, he realizado las prácticas en un centro de compensatoria (...) Al ocurrir esto, una de las medidas que se toman es que los alumnos en las materias troncales (lengua, matemáticas e inglés) reciban clases de dos docentes en una misma aula. Es por esto que siendo consciente ahora de la importancia que conlleva plantear sesiones, organizar actividades y llevarlas al aula en pareja, pienso que las parejas pedagógicas son un acierto seguro y una manera, como dije antes, de atender a la diversidad en el grupo aula. (E8GA) 
Y en este sentido, identifican un valor real en la gestión del aula desde la perspectiva del apoyo educativo, ampliando posibilidades para el desarrollo del alumnado conforme a sus necesidades e intereses.

Si bien es verdad, al extrapolarlo a secundaria y las prácticas que ya hemos comenzado, he podido observar que en las aulas donde hay dos profesores, la gestión del aula se hace de forma mucho más eficiente. Aunque no se trate de parejas pedagógicas como tal, sino más bien como apoyo dentro de una misma clase, ayuda sin duda a conseguir una educación inclusiva. (E5GA)

Desde mi punto de vista, el uso de "parejas pedagógicas" debería ser algo implementado en la enseñanza, en un principio lo consideraba sobre todo de la enseñanza secundaria, pero realmente pienso que debería ser algo llevado a cabo en todos los cursos de un centro, desde que se inicia el proceso educativo, pues como bien sabemos ya, todos y cada uno de los alumnos son un mundo, un mundo que visto desde la perspectiva de dos docentes, podría facilitar y ampliar mucho más el desarrollo y aprendizaje de cada uno de ellos. (EG6A)

Siguiendo el análisis en torno a la atención a la diversidad, descubren el valor de la pareja como proceso de observación participante que permite profundizar en lo que pasa en el aula y, en este sentido, se erigen en un instrumento al servicio de la evaluación para la comprensión.

Desde mi punto de vista como futuro docente, creo que esta estructura además de dinamizar la clase permite obtener cierta información sobre el alumnado que de otra manera no sería posible. (...)durante mi primera fase de prácticas en un centro I.E.S. he acompañado en todo momento a mi tutora a las clases y he podido observar aspectos de los alumnos y de sus explicaciones en clase que al comentarlas con ella nos dábamos cuenta de que no era consciente. (E6GD)

Relacionado con lo anterior y deteniéndonos en su sentido evaluativo, hay quien descubre en la práctica de la pareja pedagógica un proceso valioso, aunque muestra cierto miedo o preocupación a sentirse evaluado como docente por un igual, asumiendo falta de seguridad en su práctica.

A mí en un futuro también me gustaría probar la experiencia de las parejas pedagógicas, aunque pensándolo ahora, sin ninguna experiencia, quizá me sentiría cohibida al tener a otro compañero observándome y "juzgándome”. Supongo que conforme pase el tiempo y vaya ganando más seguridad como docente me atrevería, porque me gustaría atreverme aunque al principio la situación me imponga. (E2GA)

Lo que nos indican, en definitiva, las narraciones que los estudiantes hacen sobre esta estrategia, es que apostar por re-pensar la práctica educativa aportando estrategias con sentido pedagógico, como ésta, siempre es un plus; pues el aprendizaje siempre es tanto para el alumnado que lo experimenta, como para el profesorado que lo desarrolla.

\section{Discusión y conclusiones}

Para finalizar, nos gustaría destacar como este trabajo muestra cómo la enseñanza no tiene por qué ser una actividad individual, apostando por poner en acción distintos tipos de co-ense- ñanza, en concreto la de observación, de apoyo, complementaria, en estaciones y en equipos (Friend et al, 2010) en el contexto de la formación del profesorado de secundaria. Se ha podido apreciar tras los análisis, como la articulación de parejas pedagógicas recibe una valoración positiva de los- estudiantes del MAES ya que, como propuesta didáctica enriquece tanto el contenido de su formación, como la gestión del aula, la diversidad y la convivencia. En este sentido, sus apreciaciones coindicen con las aportaciones que la co-enseñanza realiza en otros contextos formativos y en los distintos niveles (Bauwens, Hourcade \& Friend, 1998; Bekerman, \& Dankner,2010). Así, el desarrollo de parejas pedagógicas permite aspectos operativos muy interesantes como gestionar grupos-clase, la recepción de feed-back inmediato, la atención simultáneamente al grupo, etc.

Otro elemento que emerge como significativo es que son capaces de proyectarlas hacia su propia práctica, hacia quienes quieren ser como docentes y cómo quieren estar en la docencia. A través de sus narraciones evidencian inquietud ante la vivencia de experiencias formativas de naturaleza distinta a la que el sistema educativo les tiene acostumbrados, como es el caso de la enseñanza colaborativa $y$, en concreto, la pareja pedagógica. Han expresado miedos fruto tanto de la vivencia de la experiencia en sí, como a su posibilidad de proyección. Otros trabajos (Salamanca 2015) apuntan en la misma dirección y naturalizan esta emoción como una parte del proceso de construcción de la identidad profesional.

La experiencia ha permitido que los-as estudiantes identifiquen algunas claves en el desarrollo de parejas pedagógicas (Cook y Friend, 1995) y que podemos concretar en:

- La necesidad de coordinación entre quienes abordan la experiencia. Si no se cuida este aspecto, se puede tener la sensación de que se trata puramente de la suma de dos partes o acciones inconexas, lo que da lugar a que el desarrollo de la estrategia pierda su sentido.

- La formación de la pareja no puede ser arbitraria, sino, que ha de ser pensada desde la complicidad entre los miembros, ya que ásta es un ingrediente fundamental. La complicidad se traduce, pedagógicamente, en un interés por trabajar juntos-as y buscar puntos de encuentro y enriquecimiento mutuo.

- La pareja pedagógica implica, por lo menos, la intencionalidad de descubrirse mutuamente como primer paso para construir conjuntamente.

- La necesidad de definición de roles equilibrados en el aula. Obviar esto puede llevar a la existencia de una posición dominante frente a otra, generar conflictos dentro de la propia pareja o proyectar una imagen errónea. Pensar en los turnos de intervenciones o formas de complementarse es fundamental, pues las aportaciones constantes andamiando los argumentos de otros, redunda de manera negativa en el seguimiento del discursos y en la profundización en los mismos. El eje en el que debe pivotar el proceso, centrado en el alumnado, puede verse desplazado por el protagonismo que adopten los discursos docentes.

Para concluir, podemos afirmar como lo valioso de la experiencia es cómo se vive que la pareja pedagógica puede ser un dispositivo para rediseñar los procesos y los contextos educativos en los que conviven, permitiendo hacer otras cosas, y es otras formas de hacer tienen que ver con la educación inclusiva: desarrollar propuestas cooperativas, ofrecer espacios de diálogo e intercambio, acompañar procesos, redefinir roles etc. Así, las 
parejas pedagógicas pueden ser consideradas como una palanca a la inclusión_(Ainscow, 2004) que posibilite, no solo imaginar, sino también construir otro modelo de escuela.

\section{Referencias bibliográficas}

Ainscow, M. (2004). El desarrollo de sistemas educativos inclusivos: ¿ Cuáles son las palancas de cambio. Journal of educational change, 5(4), 1-20.

Bekerman, D. G., \& Dankner, L. A. (2010). La pareja pedagógica en el ámbito universitario, un aporte a la Didáctica Colaborativa. Formación universitaria, 3(6), 3-8.

Bolivar, A. (2007). La formación inicial del profesorado de secundaria y su identidad profesional. ESE. Estudios sobre educación, 12, 13-30.

Bruffee, K. A. (1999). Collaborative learning: Higher education, interdependence, and the authority of knowledge. Baltimore: Johns Hopkins University Press.

Carbonell, J. (2001). La aventura de innovar. El cambio en la escuela. Madrid. Morata.

Cook, L., \& Friend, M. (1995). Co-teaching: Guidelines for creating effective practices. Focus on exceptional children, 28(3), $1-16$.

Chanmugam, A. y Gerlach, B. (2013). A Co-Teaching Model for Developing Future Educators' Teaching Effectiveness. International Journal of Teaching and Learning in Higher Education, 25(1), 10-117.

Dieker, L. (2013).Desmitifying Secondary Inclusion: powerful schoolwide \& classrooms strategies. New York. Dude Publishing.

Dieker, L. A., \& Murawski, W. W. (2003). Co-teaching at the secondary level: Unique issues, current trends, and suggestions for success. The High School Journal, 86(4), 1-13.

Durán, D., \& Miquel, E. (2003). Cooperar para enseñar y aprender. Cuadernos de pedagogía, 331, 73-76.

Friend, M., \& Cook, L. (2007). Interactions: Collaboration skills for school professionals. Boston: Pearson Education Inc.

Friend, M., Cook, L., Hurley-Chamberlain, D. \& Shamberger, C. (2010). Co-Teaching: an illustration of the complexity of collaboration in special education. Journal of Educational and Psychological Consultation, 20 (2), 9-27.

Friend, M., Reising, M., \& Cook, L. (1993). Co-teaching: An overview of the past, a glimpse at the present, and considerations for the future. Preventing School Failure: Alternative Education for Children and Youth, 37(4), 6-10.

García, M. \& García, M. J. (2015). El aprendizaje y servicio en la formación inicial del profesorado: de las prácticas educativas críticas a la institucionalización curricular. Profesorado: Revista de Currículum y Formación del Profesorado, 19(1), 8-25.

Hoyuelos, A. (2004). La pareja educativa: un reto cultural. Infancia, 86, 4-10.
Keefe, E. \& Moore, V. (2004). The Challenge of Co-Teaching in Inclusive Classrooms at the High School Level: What the Teachers Told Us. American Secondary Education 32 (3), 77-88.

Kroegera, S., Embury, D., Cooper, A., Brydon-Miller, M., Laine, C. y Johnson, H. (2012). Stone soup: using co-teaching and photovoice to support inclusive education. Educational Action Research, 20(2), 183-200. doi: 10.1080/09650792.2012.676285

Martínez Bonafé, J. (2010). Aprender el oficio docente sistematizando la práctica. En Pérez Gómez, A. (coord.). Aprender a enseñar en la práctica: procesos de innovación y prácticas de formación en Educación Secundaria (Pp. 107-120). Barcelona: Grao.

Moriña, A. \& Parrilla, A. (2006). Criterios para la formación permanente del profesorado en el marco de la educación inclusiva. Revista de Educación, 339, 517-539.

Murawski, W. W., \& Lee Swanson, H. (2001). A meta-analysis of co-teaching research: Where are the data?. Remedial and special education, 22(5), 258-267.

Parrilla (2003) La voz de la experiencia: la colaboración como estrategia de inclusión. Aula de Innovación Educativa, 121, 43-48.

Noddings, N. (2002). Educating moral people. A caring alternative to character education. New York: Teachers College Press.

Pugach, M. C., \& Johnson, L. J. (2002). Collaborative practitioners, collaborative schools. Love Publishing Company, 9101 E. Kenyon Ave., Suite 2200, Denver, CO 80237.

Rodríguez, F. (2014). La co-enseñanza, una estrategia para el mejoramiento educativo y la inclusión. Revista latinoamericana de educación inclusiva, 8(2), 219-233.

Rodríguez, E., \& Grilli, J. (2013). La pareja pedagógica: Una estrategia para transitar y aprender el oficio de ser profesor. Páginas de Educación, 6(1), 61-81.

Salamanca, C. (2015). Dos docentes, dos miradas: la pareja educativa. Tarbiya, revista de Investigación e Innovación Educativa, (42).

Scruggs, T. E., Mastropieri, M. A., \& McDuffie, K. A. (2007). Co-teaching in inclusive classrooms: A metasynthesis of qualitative research. Exceptional Children, 73(4), 392-416.

Sierra, J. E.; Caparrós, E. \& Martín, Ma A. (2016). Cultivar la convivencia cuidando las relaciones. Reflexiones desde la práctica educativa con menores en protección. En Castilla, $\mathrm{M}^{\mathrm{a}} \mathrm{T}$.; Leiva, J.; Martín, V. y Vila, E. (Coords.). Formación y perfil de los profesionales para la mejora de la convivencia en contextos sociales y educativos (pp. 47-56). Barcelona: Wolters Kluwer.

Stainback, S. y Stainback. W. (1999) (Coords): Aulas Inclusivas. Madrid: Narcea.

Suárez-Díaz, G. (2016). Co-enseñanza: concepciones y prácticas en profesores de una Facultad de Educación en Perú. Revista Electrónica de Investigación Educativa, 18(1), 166-182. Recuperado de http://redie.uabc.mx/redie/article/view/786

Wather-Tomas. C., Bryant,M. \& Land, S. (1996). Planning for Effective Co-Teaching The Key to Successful Inclusion. The Key to Successful Inclusion. Remedial and Special Education 17(4), 255-264. 